\title{
Comparison of the Effectiveness of Hydroxyethyl Starch (Voluven) Solution With Normal Saline in Hemorrhagic Shock Treatment in Trauma
}

\author{
Kambiz Masoumi ${ }^{\mathrm{a}}$, Arash Forouzan ${ }^{\mathrm{a}, \mathrm{b}}$, Ali Asgari Darian ${ }^{\mathrm{a}}$, Alireza Rafaty Navaii ${ }^{\mathrm{a}}$
}

\begin{abstract}
Background: Appropriate fluid therapy affects morbidity and mortality rates. A conclusion is yet to be reached on the role of crystalloids and colloids in immediate fluid therapy. This study was done to determine the suitable solution in immediate resuscitation of patients with hemorrhagic shock caused by tissue trauma.

Methods: One hundred trauma patients with hemorrhagic shock, who underwent fluid therapy in the emergency unit, were assigned randomly to two groups of hydroxyethyl starch (Voluven) and normal saline. Before and after fluid therapy, $1 \mathrm{cc}$ of blood was taken from all patients in order to determine and compare base excess levels.
\end{abstract}

Results: In hydroxyethyl starch (Voluven) and normal saline groups, base excess level after solution therapy increased about 9.65 and 5.46 volumes, respectively, in which augmentation in hydroxyethyl starch (Voluven) group is significantly higher than normal saline group ( $\mathrm{P}$ $\leq 0.001)$.

Conclusion: By using hydroxyethyl starch (Voluven) for fluid therapy in hemorrhagic shock caused by trauma, serum base excess decreases and results in improvement in tissue perfusion and better balance in acid-base status and it seems to be superior over normal saline administration, but the building block of the ideal fluid therapy should still remain with the physician's final clinical judgment.

Keywords: Trauma; Hemorrhagic shock; Fluid therapy; Normal saline; Hydroxyethyl starch; Colloidal solution

\section{Introduction}

Trauma is the fourth leading cause of death in the world and the most common cause of referral to the emergency ward.

Manuscript accepted for publication August 18, 2016

aDepartment of Emergency Medicine, Imam Khomeini General Hospital, Ahvaz Jundishapur University of Medical Sciences, Ahvaz, Iran

${ }^{b}$ Corresponding Author: Arash Forouzan, Department of Emergency Medicine, Imam Khomeini General Hospital, Ahvaz Jundishapur University of Medical Sciences, Ahvaz, Iran. Email: md_89864@yahoo.com

doi: http://dx.doi.org/10.14740/jocmr2702w
Approach to shock in traumatic patients is of critical importance [1-3]. Fluid therapy is an important issue in shock resuscitation and its main purpose is providing adequate intravenous fluids for maintaining intravascular volume and cardiac output, oxygen-carrying capacity, and sequentially sustaining tissue perfusion, correcting coagulopathy, acid-base balance and electrolytes [1]. Intravenous fluids used in resuscitation are divided to two basic groups of crystalloids and colloids. Although many researches have been done on the principles of fluid therapy in hemorrhagic shock and effects of different therapeutic approaches on the side effects of shock, discussions about exact role of crystalloids and colloids in immediate fluid therapy still remain unresolved [2].

Resuscitation with crystalloid fluids dilutes plasma proteins and results in decreased oncotic pressure which leads to edema in gastrointestinal or respiratory system $[4,5]$. Although administrating it in acute hemorrhage results in faster improvement of filling pressure, arterial blood pressure and heart rate. Crystalloids are more available and cheaper compared to colloids but due to some challenges in their administration such as high volumes requirement, durability, fluid accumulation, edema and electrolyte disorders, a new approach in applying colloids has emerged. Colloids are required in volumes equal to blood volume losses and have greater and quicker effect in preventing hypotension over crystalloids $[1,2,4,5]$.

Hypoperfusion has been addressed as the predictor of mortality in shock [6] and is determined by the value of base deficit. Base deficit refers to the amount of base in mili-mol required for titrating $1 \mathrm{~L}$ of whole blood to reach a $\mathrm{PH}$ of 7.4 in terms of fully oxygenated blood at a temperature of $37^{\circ} \mathrm{C}$ and a $\mathrm{pCO}_{2}$ of $40 \mathrm{~mm} \mathrm{Hg}$ [3]. This index is typically calculated through analyzing arterial blood gasses and the normal range is between 0 and 2 [7]. Studies have shown the mortality rate of traumatic patients is predictable via their base excess value during the first $24 \mathrm{~h}$ after the traumatic event. Most traumatic patients with hemorrhagic shock have severe base deficit [8]. Using the base deficit index in evaluating patients with trauma has great advantages especially in assessing shock volume, resuscitation efficiency and organizing therapeutic resources [911]. By calculating base deficit index at admission to the emergency ward, patients with high risk can be identified promptly and put under intensive care.

Calculation of base excess status was used in this study in order to compare Voluven impact as a colloid fluid and normal saline as a crystalloid in providing oxygen-carrying capacity and sustaining intravascular volume $[3,12,13]$. Results indi- 
cated a more rapid decrease in plasma lactate concentration, decreased fluid volume for shock resuscitation and better improvement is acid-base status during the first $24 \mathrm{~h}$ after treatment in patients resuscitated with Voluven. The main purpose of fluid therapy is maintaining intravascular volume and cardiac output, increasing oxygen-carrying capacity, electrolytes balance, improving surgery outcomes, reducing morbidity and mortality particularly preventing organ failure, acid-base balance and reducing recovery time after resuscitation.

\section{Methods}

\section{Study design}

This clinical trial was conducted on traumatic patients with a hemorrhagic shock diagnosis who were referred to the Emergency Medicine Unit of Emam Hospital affiliated with Ahvaz Jundishapur University of Medical Sciences.

\section{Inclusion and exclusion criteria}

The study included all patients with traumatic hemorrhagic shock who were referred to the Imam Khomeini Hospital in Ahvaz, Iran. Exclusion criteria were heart failure (reduced heart function with clinical symptoms: orthopnea, exertional dyspnea, MET $<4$ and $\mathrm{EF}<50 \%$ ); patients who received blood before the study completed; patients who died before completing the study; sensitivity to serum; transfer to the operating room before the study completed; hepatic insufficiency (decreased liver function with decreased albumin, increased PT, clinical and liver enzymes could be increased more than five times); respiratory failure (lung function decline with increasing $\mathrm{PCO}_{2}>50$ or drop in $\mathrm{PO}_{2}<60$ or observed clinical signs of respiratory distress); renal impairment: decreased renal function (GFR $<60 \mathrm{~mL} / \mathrm{min} / 1.73 \mathrm{~m}^{2}$ for at least 3 months, or significant proteinuria); sepsis: the presence of systemic inflammatory response syndrome (SIRS) with prove microbial etiology; severe anemia $(\mathrm{Hb}<10)$; patients who were considered for the diagnosis of non-hemorrhagic shock; and patients with a history of sensitivity to the solutions used in this study.

\section{Method and intervention}

Patients were evaluated for shock type. The bleeding site was investigated if hemorrhagic shock was confirmed. Exclusion criteria were age under 16, clinical and paraclinical evidence of heart failure, blood transfusion, adverse reaction to solution infusion, transfer to the operating room, clinical and paraclinical evidence of respiratory failure, clinical and paraclinical evidence of renal failure, sepsis, severe anemia $(\mathrm{HB}<10)$, and patients with other types of shock such as cardiogenic and neurogenic shock. Random allocation of patients was performed by random binary codes already devised by the physician in charge of implementation of the design. In this study, 100 patients were randomly divided into two groups using the random digit table method. At the beginning of the study, a sample of arterial blood gas (ABG) was obtained from all patients. Patients in the intervention group received $1.5 \mathrm{~L}$ of normal saline and $0.5 \mathrm{~L}$ of Voluven solution and the control group received only $2 \mathrm{~L}$ of normal saline. Again after $1 \mathrm{~h}$, ABG sample was taken and patient samples were compared in terms of deficiencies. Besides, the patient data were collected by means of a questionnaire.

\section{Outcome measures}

Primary outcome included base excess that was measured before and after fluid therapy by drawing a sample of ABG. Secondary outcome was shock index, which was assessed after serum therapy considering the condition that if the shock index (heart rate/systolic blood pressure) was greater than 1 is considered as positive.

\section{Ethical consideration}

The study was conducted after approval by the Ethics Committee of Ahvaz Jundishapur University of Medical Sciences and receiving the ethics code number IRCT201405114190N3 and obtaining consent from patients or patients' next of kin.

\section{Statistical analysis}

Since the amount of the base excess for each patient was measured before the intervention and it might be effective in treatment response, covariance analysis and restored linear methods were used for comparing Voluven effect. After data collection, the data processing was performed using SPSS software.

\section{Results}

Of 100 participants in the study, five subjects $(10 \%)$ in hydroxyethyl starch group (Voluven) and seven (14\%) in normal saline group were excluded from the study due to death, blood transfusion, and transfer to the operating room and their info was not included in the final analysis. Besides, in neither of the two groups, adverse reaction to the received solutions was observed. From a total of 88 patients participating in this study, 71 subjects $(80.6 \%)$ were male and 17 subjects $(19.3 \%)$ were female. The mean age of all the patients was $28(46 \pm 11.56)$. The mean age in Voluven group was $48(29.61 \pm 13)$ and in the normal saline group 27 (28 \pm 9.13$)$. The minimum age of participants in this study was 16 and the maximum age was 80. Comparing the age and sex of the patients, no significant difference was seen.

Mean level of base excess before fluid therapy in Voluven group was $76(1.56 \pm 0)$ and in normal saline group was $33(0.6$ $\pm 0.33)$. Given the statistical analysis, there was no significant difference between average levels of base excess before fluid therapy in both groups $(\mathrm{P}=0.082)$. The mean level of serum 


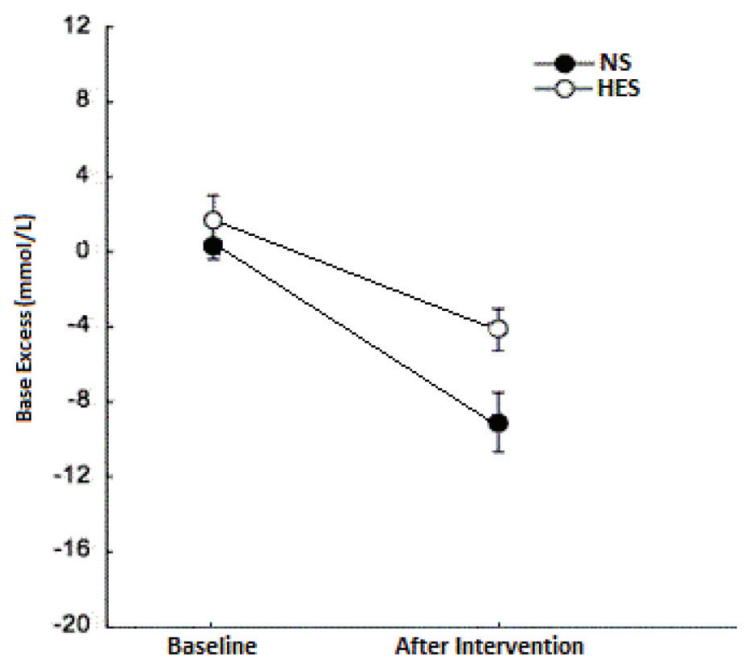

Figure 1. Base excess changes in patients with hemorrhagic shock resulting from trauma treated with normal saline hydroxyethyl starch before and after fluid therapy.

base excess after fluid therapy in Voluven group reached to 54 $(-3.9 \pm 0)$ and in normal saline reached to $9(-9 \pm 0)$. Given the statistical analysis, there was a significant difference between average base excess serum after fluid therapy between the two groups $(\mathrm{P} \leq 0.001)$. Base excess serum level in Voluven group after fluid therapy was obviously higher. The mean difference between base excess levels in Voluven group before and after fluid therapy was 5.46 which indicated a significant difference. The mean difference between the base excess serums in normal saline group was 9.65 before and after fluid therapy. A significant difference between the mean serum level of base excess fluid was observed in these groups before and after fluid therapy (Fig. 1, $\mathrm{P} \leq 0.001$ ).

\section{Discussion}

In an attempt to resuscitate patients with hemorrhagic shock, the first step includes providing therapeutic solution and immediate fluid replacement [14-17]. Fluids replacement consists of two major groups of crystalloids and colloids, the conflicting discussions of which exist surrounding their advantages and disadvantages.

In our study, the effect of hydroxyethyl starch (Voluven) solution and normal saline in shock resuscitation of traumatic patients through comparison between base excess amounts before and after resuscitation was studied. Distribution of all variables (age, sex, and base deficit) was normal before starting fluid resuscitation and there was no significant difference among the primary indexes of both groups. Duration of resuscitation between the two groups also had no significant difference, thus for the final assessment of data, we used parametric statistical tests. The end results showed that hydroxyethyl starch (Voluven) in comparison with normal saline improves base excess amount significantly.

In a study the impact of colloid solutions like Hartmann and hextend solution with solutions based on normal saline in providing a metabolic environment and tissue oxygenation perfusion was compared. The outcome showed base excess in normal saline group had a great decrease compared to colloid group. Dr. Fiz et al's study approved our study results. That study compared the effectiveness of fluid therapy with Voluven versus normal saline on 67 patients with deep trauma. In that study, contrary to our study, lactate concentration level was used as an index for shock resuscitation.

Arguments in favor of crystalloids state that in consuming colloidal solutions, albumin molecules freely enter the pulmonary interstitial space and then are washed away by the lymphatic system and return to the systemic circulation. Thus, more albumins only increase albumin accumulation that is cleared off through lymphatic system. Isotonic crystalloid fluids are effective augmentation of plasma volume for resuscitation. Crystalloid advocates argue that crystalloids are cheap and their side effects are rare. Non-existence of allergic reactions, coagulopathy, and tissue accumulation are the advantages of crystalloids. In contrast, arguments in favor of colloids explain that oncotic pressure of plasma proteins in revival with crystalloid solutions leads to filtered fluids from intravascular to the interstitial space leading to edema in the digestive system. The administration of colloids after acute hemorrhage results in faster improvement of filling pressures, arterial blood pressure and heart rate. Colloids cause less tissue edema and faster improve microcirculation $[14,15,18,19]$. Colloids cause a faster increase in intravascular volume but do not have much of a positive effect on tissue perfusion and microcirculation of vital organs of the body and there is no justifiable reason for their routine use. Precautions must be taken into account for colloid usage and in some cases their use should be avoided. Hydroxyethyl starch is a colloidal solution and today there are concerns about its impact on coagulation and renal function [16].

In a study it was demonstrated that hydroxyethyl starch administration (HES) compared with normal saline in reviving critically ill patients in intensive care unit, obviously increased the risk of acute kidney injury (AKI) development. The study recommended that the use of HES should be avoided in patients with severe sepsis and in other critically ill patients at risk for AKI [17]. Although a randomized controlled trial by Kasper et al in 2004 on trauma, burns, and postoperative patients showed no evidence of a decrease in the risk of death with colloids resuscitation compared with crystalloids [17]. Moreover, some studies claimed that despite the fact that colloids have a greater effect on increasing intravascular volume than crystalloids, but considering prognosis, neither has an advantage over the other [20]. In a study published in 1979 by Virgilio et al, resuscitations of 29 patients undergoing aortic surgery with saline fluid balance and serum containing protein were compared and it was demonstrated that despite significant differences in central venous pressure and pulmonary artery wedge pressure, pulmonary edema was similar in the two groups [11].

According to several studies conducted throughout the world on the effects of colloids and crystalloids on providing bodily needs, maintaining the balance of fluids and electrolytes, maintaining adequate perfusion of organs, maintaining proper circulation volume and homeostasis, adequate oxygen-carrying capacity, reduced morbidity and mortality during surgery 
procedures, trauma and burns and avoiding organ failure, etc, and also according to the results of our study in which application of colloids (Voluven) resulted in reduction of serum base deficit as a determinant index of tissue perfusion and providing favorable physiologic condition compared with colloids, it seems using colloids (Voluven) in resuscitating patients is preferable over crystalloids (normal saline), but it should still be noted that there is no best solution for resuscitation and fluid preservation and that basis for the ideal fluid therapy should still remain with the physician's clinical judgment.

\section{Acknowledgments}

This study is part of thesis for Alireza Rafaty Navaii. We wish to express our thanks to all our colleagues in Emergency Medicine Department of Golestan Hospital, Ahvaz Jundishapur University of Medical Sciences.

\section{References}

1. Dutton RP. Current concepts in hemorrhagic shock. Anesthesiol Clin. 2007;25(1):23-34, viii.

2. Moss GS, Gould SA. Plasma expanders. An update. Am J Surg. 1988;155(3):425-434.

3. Wilkes NJ, Woolf R, Mutch M, Mallett SV, Peachey T, Stephens R, Mythen MG. The effects of balanced versus saline-based hetastarch and crystalloid solutions on acid-base and electrolyte status and gastric mucosal perfusion in elderly surgical patients. Anesth Analg. 2001;93(4):811-816.

4. Forget P, Lois F, de Kock M. Goal-directed fluid management based on the pulse oximeter-derived pleth variability index reduces lactate levels and improves fluid management. Anesth Analg. 2010;111(4):910-914.

5. Toraman F, Evrenkaya S, Yuce M, Aksoy N, Karabulut H, Bozkulak Y, Alhan C. (2004, April). Lactic acidosis after cardiac surgery is associated with adverse outcome. In: The heart surgery forum (Vol. 7, No. 2, pp. E155-E159). Carden Jennings Publishing Co.

6. Madjdpour C, Dettori N, Frascarolo P, Burki M, Boll M, Fisch A, Bombeli T, et al. Molecular weight of hydroxyethyl starch: is there an effect on blood coagulation and pharmacokinetics? Br J Anaesth. 2005;94(5):569-576.

7. Finfer S, Bellomo R, Boyce N, French J, Myburgh J, Norton R. A comparison of albumin and saline for fluid resuscitation in the intensive care unit. N Engl J Med. 2004;350(22):2247-2256.

8. Ranucci M, De Toffol B, Isgro G, Romitti F, Conti D, Vicentini M. Hyperlactatemia during cardiopulmonary by- pass: determinants and impact on postoperative outcome. Crit Care. 2006;10(6):R167.

9. Roberts I, Blackhall K, Alderson P, Bunn F, Schierhout G. Human albumin solution for resuscitation and volume expansion in critically ill patients. Cochrane Database Syst Rev. 2011;11:CD001208.

10. Ramanathan S, Masih A, Rock I, Chalon J, Turndorf H. Maternal and fetal effects of prophylactic hydration with crystalloids or colloids before epidural anesthesia. Anesth Analg. 1983;62(7):673-678.

11. Virgilio R, Rice C, Smith D, James D, Zarins C, Hobelmann C, Peters R. Crystalloid Vs. Colloid Resuscitation: Is One Better? A Randomized Clinical Study. Survey of Anesthesiology. 1980;24(1):1

12. Ranucci M, Isgro G, Carlucci C, De La Torre T, Enginoli S, Frigiola A. Central venous oxygen saturation and blood lactate levels during cardiopulmonary bypass are associated with outcome after pediatric cardiac surgery. Crit Care. 2010;14(4):R149.

13. Miller RD, Pardo M. Basics of Anesthesia Sixth edition. Saunders. 2011.

14. Myburgh JA, Finfer S, Bellomo R, Billot L, Cass A, Gattas D, Glass P, et al. Hydroxyethyl starch or saline for fluid resuscitation in intensive care. N Engl J Med. 2012;367(20):1901-1911.

15. Laxenaire MC, Charpentier C, Feldman L. [Anaphylactoid reactions to colloid plasma substitutes: incidence, risk factors, mechanisms. A French multicenter prospective study]. Ann Fr Anesth Reanim. 1994;13(3):301-310.

16. Neff TA, Doelberg M, Jungheinrich C, Sauerland A, Spahn DR, Stocker R. Repetitive large-dose infusion of the novel hydroxyethyl starch $130 / 0.4$ in patients with severe head injury. Anesth Analg. 2003;96(5):1453-1459, table of contents.

17. Kasper SM, Meinert P, Kampe S, Gorg C, Geisen C, Mehlhorn U, Diefenbach C. Large-dose hydroxyethyl starch 130/0.4 does not increase blood loss and transfusion requirements in coronary artery bypass surgery compared with hydroxyethyl starch 200/0.5 at recommended doses. Anesthesiology. 2003;99(1):42-47.

18. Perel P, Roberts I, Ker K. Colloids versus crystalloids for fluid resuscitation in critically ill patients. Cochrane Database Syst Rev. 2013;2:CD000567.

19. So KW, Fok TF, Ng PC, Wong WW, Cheung KL. Randomised controlled trial of colloid or crystalloid in hypotensive preterm infants. Arch Dis Child Fetal Neonatal Ed. 1997;76(1):F43-46.

20. Ellger B, Freyhoff J, Van Aken H, Marcus MAE, Booke M. High dose volume replacement using HES 130/0.4 during major surgery does not alter coagulation. Eur J Anaesthesiol, 2002;19(Suppl 24):77. 